\title{
REVIEW
}

\section{Year in review 2010: Critical Care - neurocritical care}

\author{
Michael T Scalfani and Michael N Diringer*
}

\begin{abstract}
In 2010 Critical Care published a large number of articles on critical care aspects of neurologic and neurosurgical conditions. These aspects included investigation of diagnostic criteria for bacterial meningitis, critical illness myopathy and their relationship to systemic inflammation. A number of studies investigated the biology of sepsis-related delirium, its biomarkers, its relationship to inflammation and its impact on outcome. Other teams reported on the use of magnetic resonance imaging, biomarkers and electroencephalogram to predict outcome in patients who were comatose following cardiac arrest. Our understanding of the pathophysiology as well as management of subarachnoid hemorrhage was addressed in several papers. Topics included the effect of hemodynamic treatment of delayed cerebral ischemia, pulmonary edema and the impact of subarachnoid hemorrhage on endocrine function. Finally, outcome from neurocritical care and patients' retrospective willingness to consent to the treatment they received were reported.
\end{abstract}

Several important contributions to the field of neurocritical care were published in Critical Care during 2010. These articles can be gathered into six key areas: diagnostic criteria, delirium and encephalopathy, predicting neurologic outcome after cardiac arrest, subarachnoid hemorrhage $(\mathrm{SAH})$ and outcome from neurocritical care.

\section{Diagnostic criteria Meningitis}

Favorable outcome from bacterial meningitis requires rapid diagnosis and immediate initiation of antibiotic

*Correspondence: diringerm@neuro.wustl.edu

Neurology/Neurosurgery Intensive Care Unit, Department of Neurology,

Washington University School of Medicine, Campus Box 8111, 660 S. Euclid Avenue, St Louis, MO 63110, USA therapy [1], yet distinguishing between bacterial and nonbacterial meningitis can sometimes prove difficult. The use of cerebrospinal fluid (CSF) lactate, as opposed to conventional tests (such as CSF glucose, CSF/plasma glucose ratio, CSF protein concentration, and CSF leukocyte count), has been investigated in a number of studies to distinguish between bacterial and nonbacterial meningitis.

Huy and coworkers performed a literature review and meta-analysis to evaluate the usefulness of CSF lactate concentration for this purpose [2]. From the 25 studies they identified, the authors concluded that CSF lactate alone had a high degree of accuracy in distinguishing between bacterial and nonbacterial meningitis and performs better than the conventional tests routinely used. CSF lactate was found to be less useful if its concentration was low, but when elevated it was helpful, especially if the diagnosis was otherwise inconclusive. This suggests that any elevation in CSF lactate concentration above normal for the assay used could be employed as a diagnostic marker despite the difference in cut-off values caused by variance in methods, instruments and hospital laboratories. While the authors conclude CSF lactate is a useful marker to distinguish between bacterial and nonbacterial meningitis, it is not meant to replace conventional tests as they are necessary to diagnose meningitis. Rather, interpretation of lactate alone is a better discriminator between bacterial and nonbacterial meningitis than conventional tests. As a result, measurements of CSF lactate may be considered part of standard practice to distinguish between bacterial and nonbacterial meningitis.

\section{Critical illness myopathy}

Critical illness myopathy (CIM) frequently complicates serious illness and often results in prolonged mechanical ventilation. This complication is characterized by the atrophy of type II muscle fibers and thick filaments, as well as nonexcitable muscle membranes [3]. Risk factors for developing CIM are not well understood.

Weber-Carstens and colleagues performed a prospective observational study of 40 ICU patients with a Simplified Acute Physiology Score II $\geq 20$ to identify risk 
factors for CIM. Patients were evaluated on three consecutive days within 1 week of ICU admission [4]. The authors investigated demographics, inflammatory, metabolic and hemodynamic parameters, and medical treatment prior to the first nonelectromyography signs of CIM. On univariate analysis, inflammation, disease severity, decreased insulin sensitivity, catecholamine use and sedation requirements were all significantly associated with CIM. Low-dose hydrocortisone treatment in septic shock and administration of neuromuscular blocking agents or aminoglycosides, however, were not related to CIM. In a multivariate Cox proportional hazard regression analysis, IL-6 was the only independent risk factor for CIM (95\% confidence interval $(\mathrm{CI})=1.00$ to $1.01 ; P=0.005)$; however, the overall effect was small. The authors hypothesize that impairment of growthfactor-mediated intracellular signaling induced by systemic inflammation is important in the pathophysiology of CIM. A weakness of this study was that subject blood samples were only taken at two separate time points, which makes it impossible to determine the daily course of the inflammatory parameters.

\section{Delirium/encephalopathy}

In hospitalized patients, delirium is associated with increased mortality, length of stay and cost $[5,6]$. The epidemiology of delirium in ICUs is poorly understood, however, in part because its evaluation has been limited to single-center studies. Salluh and coworkers performed a 1-day point-prevalence study in 104 ICUs [7]. Patients were excluded if they had Glasgow Coma Scale (GCS) $<14$ from a primary neurologic diagnosis, were legally blind or deaf, could not speak the language of the country where the ICU was located, or were moribund. Of the 975 patients screened, only 497 were enrolled. Because of the use of sedative drugs, only 232 (46.6\%) of the enrolled patients could be evaluated. Of the patients evaluated, 75 met the diagnostic criteria for delirium using the Confusion Assessment Method for the ICU. The authors found delirium to be associated with higher mortality, and longer ICU and hospital length of stays. This suggests that sicker patients have a higher risk of developing delirium, as well as a higher risk of staying longer or dying in the ICU. The main modifiable risk factors were the use of invasive devices and sedatives (midazolam). This study confirmed findings from single-center studies indicating that delirium is common in ICU patients and is associated with longer length of stay and adverse outcomes. Important weaknesses of this study are the high number of patients excluded and that so many patients could not be assessed due to sedation. Hence, only $24 \%$ of those patients originally screened were evaluated for delirium. This raises an important question of whether we are sedating too many patients in the ICU.
In addition, the true incidence of delirium in the ICU is difficult to determine from this study due to the exclusion of the majority of patients.

Another important insight into delirium was reported by $\mathrm{Mu}$ and colleagues in a prospective cohort study [8]. In postoperative patients who had undergone coronary artery bypass graft surgery they measured serum cortisol levels and assessed their relationship to delirium. A total of 243 patients were assessed for delirium twice daily during the first five postoperative days using the Confusion Assessment Method for the ICU. The presence of delirium was compared with serum cortisol measured on the morning of the first postoperative day. Postoperative delirium was identified in $50 \%$ of patients $(n=123)$. A high serum cortisol level (odds ratio $(\mathrm{OR})=$ $3.09,95 \% \mathrm{CI}=1.76$ to $5.41, P<0.001)$, occurrence of complications during the first day after surgery $(\mathrm{OR}=$ $2.49,95 \% \mathrm{CI}=1.18$ to $5.21, P=0.016)$, a history of diabetes mellitus $(\mathrm{OR}=1.91,95 \% \mathrm{CI}=1.00$ to $3.62, P=$ $0.049)$, prolonged duration of surgery $(\mathrm{OR}=1.36,95 \% \mathrm{CI}$ $=1.01$ to $1.83, P=0.043)$ and increasing age $(\mathrm{OR}=1.11$, $95 \% \mathrm{CI}=1.06$ to $1.16, P<0.001)$ were all associated with increased risk of postoperative delirium. Postoperative delirium was also associated with increased length of stay and with worse outcome.

This study has some limitations. Serum cortisol levels were not measured at baseline and were measured only once after surgery. As a result the data could potentially be skewed prior to surgery by including patients who had elevated cortisol and who might have been at greater risk of developing postoperative delirium due to preexisting conditions. In addition, the investigators did not perform baseline psychiatric or cognitive screening tests. Any mental disorders present prior to surgery could influence the development of postoperative delirium.

Systemic infections can cause serious cognitive dysfunction in ICU patients, which may be related to proinflammatory cytokines. IL-1 is a likely cytokine candidate due to its influence on both the immune system as well as the central nervous system. To address this question, Terrando and colleagues investigated the development of LPS-induced cognitive dysfunction in wild-type and IL-1 knockout mice [9]. After lipopolysaccharide (LPS) challenge, performance on behavioral tests of memory and response to novel environments deteriorated and reactive microgliosis was seen in the hippocampus. In addition, plasma TNF $\alpha$, IL-1 $1 \beta$, IL-6, and high-mobility group box-1, IL- 6 and IL-1 $\beta$ mRNA all increased. Administration of IL-1 receptor antagonist significantly reduced plasma cytokines and hippocampal microgliosis, and ameliorated cognitive dysfunction; similar results were seen in IL-1 knockout mice without receiving IL-1 receptor antagonist. These data suggest that behavioral dysfunction could be prevented by 
blocking the IL-1 signal, attenuating the inflammatory cascade. Future studies would need to be carried out to determine whether responses are seen in patients.

Sepsis-associated encephalopathy (SAE) is defined as diffuse cerebral dysfunction that is caused by a systemic response to infection without any evidence of a central nervous system infection $[10,11]$. However, the pathophysiology of SAE is not well understood. In a prospective cohort study, Berg and colleagues found that brain function might be impaired during systemic inflammation due to a reduction in the plasma ratio between branched-chain amino acids and aromatic amino acids (BCAA/AAA ratio), which alters their uptake into the brain [12]. Twelve healthy adult male subjects (aged 20 to 33) were enrolled. After an overnight fast, all subjects underwent measurements of cerebral blood flow (CBF), plasma BCAA/AAA ratio, cerebral delivery, net exchange of large amino acids and ammonia level. Paired arteriojugular venous blood samples were taken at baseline and after a 4-hour continuous intravenous infusion of purified Escherichia coli LPS. This LPS infusion resulted in an increase in plasma phenylalanine and a significant decrease in the concentration of all other large neutral amino acids except for isoleucine. As the BCAA/AAA ratio decreased, there was an increase in the levels of the neurotoxic AAA phenylalanine - suggesting a relationship to encephalopathy. In this study, however, the investigators did not perform cognitive tests on the subjects. Therefore, while systemic inflammation may affect brain function by reducing the $\mathrm{BCAA} / \mathrm{AAA}$ ratio, it is difficult to draw any conclusions regarding the role of the $\mathrm{BCAA} / \mathrm{AAA}$ ratio in SAE until cognitive function is assessed.

In another study investigating the pathophysiology of SAE, Szatmári and colleagues assessed cerebrovascular reactivity using an acetazolamide challenge in SAE patients [13]. In a prospective cohort, patients with clinical sepsis and disturbances of consciousness of any degree were studied. In addition, 20 age-matched and sex-matched controls without previous diseases affecting cerebral vasoreactivity were also included. Transcranial Doppler blood flow velocities were then measured at baseline and 5, 10, 15 and 20 minutes after intravenous administration of $15 \mathrm{mg} / \mathrm{kg}$ acetazolamide. The time course of the $\mathrm{CBF}$ velocity response to acetazolamide was measured and cerebrovascular reactivity was calculated (the percentage increase in the mean blood flow velocity). In addition, the maximum vasodilatory effect (cerebrovascular reserve capacity) was determined. The authors found that the time course of the vasomotor reaction to acetazolamide was delayed and the maximal vasodilatory response was reduced in SAE patients. The authors propose that cerebral hemodynamic changes might be involved in early pathogenesis of SAE.
SAE is common in patients with septic shock and has been associated with elevated levels of brain-specific S100 $\beta$ protein. In some studies, treatment of septic patients with drotrecogin alfa (DrotAA) reduces mortality in patients with severe sepsis-induced organ failure. Spapen and coworkers sought to determine whether DrotAA would reduce S100 $\beta$ levels and sepsis-associated encephalopathy [14]. Patients with pre-existing or acute neurological disorders were excluded. Those enrolled were classified into two groups based on their level of consciousness: GCS $\geq 13$ and GCS $<13$. DrotAA was given as a continuous infusion of $24 \mu \mathrm{g} / \mathrm{kg} /$ hour for 96 hours. The level of S100 $\beta$ was measured before sedation and serially after the start of DrotAA infusion. Of the 54 patients in the study, one-half had elevated $S 100 \beta$ levels. In patients with GCS $<13$ receiving DrotAA, S100 $\beta$ levels fell from baseline at 64 and 96 hours (both $P<0.05)$. In addition, those who received DrotAA had significantly lower S100 $\beta$ levels at 64 and 96 hours compared with those who did not receive treatment. In patients with GCS $\geq 13$, however, DrotAA had no effect on $S 100 \beta$ levels. These data suggest an association between the severity of SAE and serum levels of S100 $\beta$, but the measure of encephalopathy was limited.

\section{Cardiac arrest}

Our ability to predict neurological outcome of patients who remain comatose following cardiac arrest is limited. In addition, the existing data must be re-evaluated due to advent of treatment with hypothermia. Choi and colleagues sought to determine whether diffusion-weighted magnetic resonance imaging (MRI) could improve prediction of outcome [15]. They studied 39 patients who suffered outof-hospital cardiac arrest, performing diffusion-weighted MRI within 5 days of cardiac arrest and then comparing functional outcome at 3 months. Patients were treated with hypothermia in the latter half of the study, limiting interpretation. Diffusion-weighted MRI findings were categorized into four patterns: normal, isolated cortical injury, isolated deep gray nuclei injury, and mixed injury (cortex and deep gray nuclei). Twenty-three subjects with normal MRI scans served as controls. The authors found that a mixed pattern of brain injury involving cortex and deep gray nuclei on MRI predicted an unfavorable outcome, defined as a Glasgow Outcome Scale (GOS) score of 1 to 3 .

Another approach to predicting neurological outcome of cardiac arrest patients involved the use of serum biomarkers. Steffen and colleagues investigated the ability of neuron-specific enolase to predict outcome from cardiac arrest treated with hypothermia [16]. Ninety-seven subjects were compared with 133 historical cardiac arrest patients not treated with hypothermia. In this observational prospective cohort study, serum 
neuron-specific enolase was measured 72 hours after ICU admission and the neurological outcome (Pittsburgh cerebral performance category) was recorded at ICU discharge. In univariate analysis, neuron-specific enolase serum levels were lower in patients treated with hypothermia. The 72-hour neuron-specific enolase cut-off levels, however, did not reliably predict poor neurological outcome. The authors also note that 1-year survival was significantly higher for the hypothermia group compared with the historical nonhypothermic controls.

Rossetti and coworkers focused on the use of continuous electroencephalogram (EEG) to predict outcome in cardiac arrest patients treated with hypothermia [17]. In a cohort of 34 patients, continuous EEG demonstrating nonreactive or discontinuous background during hypothermia was strongly associated with unfavorable outcome in their patients. In addition, nonreactive background EEG was 100\% predictive of mortality (95\% $\mathrm{CI}=74$ to $100 \%)$ with a false-positive rate of $0(95 \% \mathrm{CI}=$ 0 to $18 \%$ ). These data suggest that early continuous EEG findings might be a better predictor of outcome than biomarkers or imaging.

\section{Spontaneous aneurysmal subarachnoid hemorrhage}

The most common and potentially treatable cause of secondary neurological injury after aneurysmal SAH is delayed cerebral ischemia (DCI). DCI occurs several days after hemorrhage and is considered to result from reductions in $\mathrm{CBF}$ below critical ischemic thresholds. Several factors are thought to contribute to DCI including large artery vasospasm, impaired cerebral autoregulation and intravascular volume depletion [18]. Triple-H therapy (hypervolemia, hypertension, hemodilution) is the mainstay treatment of DCI with the goal of increasing cerebral perfusion pressure. Dankbaar and colleagues performed a systematic review of the literature addressing the effectiveness of triple- $\mathrm{H}$ therapy and its components on clinical outcome [19]. Eleven studies of triple- $\mathrm{H}$ therapy were identified and included in their meta-analysis. The authors concluded that hemodilution did not change CBF and that only one of the seven studies on hypervolemia showed a significant increase in $\mathrm{CBF}$. There was a significant increase in CBF in two of the four studies applying hypertension and in one study using triple-H therapy. None of these studies used control groups or randomization. The authors conclude that there is no evidence from controlled studies for a beneficial effect of triple-H therapy or its separate components on CBF in SAH patients.

The conclusion that there is no evidence to support triple- $\mathrm{H}$ therapy is misleading, however, as the treatment has not been properly studied to date. This systematic review points out the lack of well-designed studies.
Owing to the amount of underpowered and poorly designed studies, it is difficult to come to any conclusions regarding effectiveness.

In a prospective cohort study, Bendel and coworkers evaluated neuroendocrine status after SAH and its impact on clinical outcome [20]. They enrolled 30 subjects and 16 controls. The study focused on insulin-like growth factor-1 (IGF-1), which is associated with neuronal growth, cell death and apoptosis after ischemic stroke [21]. The authors found that serum IGF-1 concentrations were significantly lower in SAH patients on days 1 to $5 \mathrm{com}-$ pared with controls $(P=0.01)$. There was no significant difference at 3 months between the groups. In addition, there was no relationship between the severity of SAH and the IGF-1 concentration. The data showed patients with GOS $\leq 4$ had lower IGF-1 concentrations and lower health-related quality of life compared with patients with higher GOS scores. This is the first published study to evaluate IGF-1 in the acute phase of aneurysmal SAH. The authors suggest that low IGF-1 following SAH might be associated with poor outcome or death.

Pulmonary edema is common after SAH [22], but its management is complicated by the need to rigorously avoid hypovolemia in these patients. Hoff and colleagues prospectively studied intravascular volume (using pulse dye densitometry) in $102 \mathrm{SAH}$ patients, comparing those with and without pulmonary edema [23]. Surprisingly, those patients who developed pulmonary edema (17 out of 102) had lower mean circulating blood volume than those without. As a result, fluid management in SAH patients that develop pulmonary edema is more difficult. The authors concluded that treatment for pulmonary edema with diuresis must be weighed against the risk of worsening hypovolemia in SAH patients and thus increasing the risk of DCI.

\section{Outcome from neurocritical care}

Despite advances in diagnosis and treatment, patients admitted to a neurocritical care unit have poor functional outcome and high mortality [24]. In a retrospective review of 796 consecutive patients admitted to a nonsurgical neurocritical care unit, Kiphuth and colleagues found an in-hospital mortality of $22.5 \%$. Good long-term functional outcome (determined by the modified Rankin Scale) was seen in only $28.4 \%$ [25]. Variables found to be independently associated with outcome were admission diagnosis, age, duration of mechanical ventilation and Therapeutic Intervention Scoring System-28 on day 1. When diagnoses were compared, the authors found that patients with Guillain-Barré syndrome and myasthenia gravis had a good long-term outcome more often than those with other diagnoses.

In another study, Kiphuth and colleagues investigated the relationship between functional outcome and 
retrospective agreement to neurocritical care [26]. The purpose of this study was to determine whether retrospective consent to neurocritical care was influenced by functional outcome. In this study of 704 consecutive patients admitted to a nonsurgical neurocritical care unit, $91 \%$ of patients who were independent at 1 year would again agree to the care they received. Only $19 \%$ of those who were dependent for activities of daily living, however, would have agreed retrospectively to neurocritical care. The authors conclude that efforts must be taken to increase public awareness regarding the importance of advanced directives, and further research must focus on determining highly specific and sensitive predictors for outcome in these patients.

In patients suffering from nontraumatic supratentorial intracerebral hemorrhage, the influence of anemia on functional outcome and mortality was investigated by Diedler and colleagues. In a retrospective cohort study of 196 patients, $23.5 \%$ had a favorable outcome (moderate disability or better) [27]. The mean hemoglobin concentration was significantly lower (12.3 versus $13.7 \mathrm{~g} / \mathrm{dl}$, $P<0.001)$ in those with poor outcome. On multivariate analysis, the independent predictors of poor outcome at 90 days were mean hemoglobin, age and NIH Stroke Scale score on admission. The authors conclude, however, that they could not determine whether anemia causes further brain injury or is a marker of severe injury. Despite the difference in mean hemoglobin in those patients with poor outcome, this is of uncertain clinical significance.

Outcome after traumatic brain injury is difficult to predict. Recent investigations have looked into a variety of biomarkers to better predict and monitor outcome after brain trauma. Biomarkers investigated include glial fibrillary acidic protein [28], neurofilament light chain [29], S100 $\beta$ [30], tissue transglutaminases [31], ubiquitin [32], $\beta$-amyloid precursor proteins [33] and tau [34]. Dong and colleagues investigated the use of the biomarker resistin predicting outcome after traumatic brain injury. This group previously reported that elevated levels of resistin are found in blood of patients with intracerebral hemorrhage and that higher levels were associated with poor outcome. The authors subsequently studied a cohort of 94 patients with severe traumatic brain injury and compared their resistin levels with those of 50 healthy controls [35]. Plasma resistin levels were elevated during the initial 6 hours after injury and peaked within 24 hours; the patients' levels were substantially higher than those of the healthy controls. Plasma resistin levels were negatively correlated with GCS $(P<0.001)$ and were an independent predictor of 1-month survival. The data suggest that the plasma resistin level might reflect the initial brain injury and could become a useful biomarker to predict survival in these patients.

\section{Conclusion}

In 2010 the field of neurocritical care received increased attention, with an increasing number of articles being published in Critical Care. The issues of diagnostic criteria for meningitis and critical illness myopathy were further investigated. In addition, studies of the epidemiology, risk factors, and treatment for delirium and encephalopathy in the ICU were published. Aspects of cardiac arrest, aneurysmal SAH and outcomes in neurocritical care were re-addressed using new approaches. Overall, there were many quality articles published in Critical Care during 2010 that have added to our scientific and clinical knowledge in the field of neurocritical care.

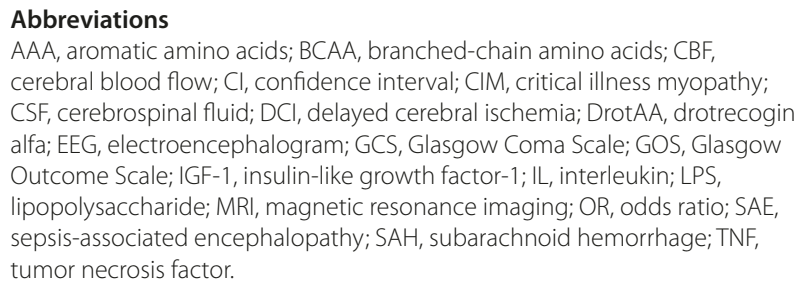

\section{Competing interests}

The authors declare that they have no competing interests.

Published: 5 December 2011

\section{References}

1. Saez-Llorens X, McCracken GH, Jr: Bacterial meningitis in children. Lancet 2003, 361:2139-2148

2. Huy NT, Thao NT, Diep DT, Kikuchi M, Zamora J, Hirayama K: Cerebrospinal fluid lactate concentration to distinguish bacterial from aseptic meningitis: a systemic review and meta-analysis. Crit Care 2010, 14:R240.

3. Stevens RD, Marshall SA, Cornblath DR, Hoke A, Needham DM, de Jonghe B, Ali NA, Sharshar T: A framework for diagnosing and classifying intensive care unit-acquired weakness. Crit Care Med 2009, 37(10 Suppl):S299-S308.

4. Weber-Carstens S, Deja M, Koch S, Spranger J, Bubser F, Wernecke KD, Spies $C D$, Spuler S, Keh D: Risk factors in critical illness myopathy during the early course of critical illness: a prospective observational study. Crit Care 2010, 14:R119.

5. Ouimet S, Kavanagh BP, Gottfried SB, SkrobikY: Incidence, risk factors and consequences of ICU delirium. Intensive Care Med 2007, 33:66-73.

6. Thomason JW, Shintani A, Peterson JF, Pun BT, Jackson JC, Ely EW: Intensive care unit delirium is an independent predictor of longer hospital stay: a prospective analysis of 261 non-ventilated patients. Crit Care 2005, 9:R375-R381

7. Salluh JI, Soares M, Teles JM, Ceraso D, Raimondi N, Nava VS, Blasquez P, Ugarte S, Ibanez-Guzman C, Centeno JV, Laca M, Grecco G, Jimenez E, ÁriasRivera S, Duenas C, Rocha MG, Delirium Epidemiology in Critical Care Study Group: Delirium epidemiology in critical care (DECCA): an international study. Crit Care 2010, 14:R210.

8. Mu DL, Wang DX, Li LH, Shan GJ, Li J, Yu QJ, Shi CX: High serum cortisol level is associated with increased risk of delirium after coronary artery bypass graft surgery: a prospective cohort study. Crit Care 2010, 14:R238.

9. Terrando N, Rei Fidalgo A, Vizcaychipi M, Cibelli M, Ma D, Monaco C, Feldmann M, Maze M: The impact of IL-1 modulation on the development of lipopolysaccharide-induced cognitive dysfunction. Crit Care 2010, 14:R88.

10. Wilson JX, Young GB: Progress in clinical neurosciences: sepsis-associated encephalopathy: evolving concepts. Can J Neurol Sci 2003, 30:98-105.

11. Piazza O, Russo E, Cotena S, Esposito G, Tufano R: Elevated S100B levels do not correlate with the severity of encephalopathy during sepsis. $\mathrm{Br} J$ Anaesth 2007, 99:518-521.

12. Berg RM, Taudorf S, Bailey DM, Lundby C, Larsen FS, Pedersen BK, Moller K: 
Cerebral net exchange of large neutral amino acids after lipopolysaccharide infusion in healthy humans. Crit Care 2010, 14:R16.

13. Szatmari S, Vegh T, Csomos A, Hallay J, Takacs I, Molnar C, Fulesdi B: Impaired cerebrovascular reactivity in sepsis-associated encephalopathy studied by acetazolamide test. Crit Care 2010, 14:R50.

14. Spapen H, Nguyen DN, Troubleyn J, Huyghens L, Schiettecatte J: Drotrecogin alfa (activated) may attenuate severe sepsis-associated encephalopathy in clinical septic shock. Crit Care 2010, 14:R54.

15. Choi SP, Park KN, Park HK, Kim JY, Youn CS, Ahn KJ, Yim HW: Diffusionweighted magnetic resonance imaging for predicting the clinical outcome of comatose survivors after cardiac arrest: a cohort study. Crit Care 2010, 14:R17.

16. Steffen IG, Hasper D, Ploner CJ, Schefold JC, Dietz E, Martens F, Nee J, Krueger A, Jorres A, Storm C: Mild therapeutic hypothermia alters neuron specific enolase as an outcome predictor after resuscitation: 97 prospective hypothermia patients compared to 133 historical non-hypothermia patients. Crit Care 2010, 14:R69.

17. Rossetti AO, Urbano LA, Delodder F, Kaplan PW, Oddo M: Prognostic value of continuous EEG monitoring during therapeutic hypothermia after cardiac arrest. Crit Care 2010, 14:R173.

18. Diringer MN: Management of aneurysmal subarachnoid hemorrhage. Crit Care Med 2009, 37:432-440

19. Dankbaar JW, Slooter AJ, Rinkel GJ, Schaaf IC: Effect of different components of triple- $\mathrm{H}$ therapy on cerebral perfusion in patients with aneurysmal subarachnoid haemorrhage: a systematic review. Crit Care 2010, 14:R23.

20. Bendel S, Koivisto T, Ryynanen OP, Ruokonen E, Romppanen J, Kiviniemi V, Uusaro A: Insulin like growth factor-l in acute subarachnoid hemorrhage: a prospective cohort study. Crit Care 2010, 14:R75.

21. Kooijman R, Sarre S, Michotte Y, De Keyser J: Insulin-like growth factor I: a potential neuroprotective compound for the treatment of acute ischemic stroke? Stroke 2009, 40:e83-e88.

22. Muroi C, Keller M, Pangalu A, Fortunati M, Yonekawa Y, Keller E: Neurogenic pulmonary edema in patients with subarachnoid hemorrhage. J Neurosurg Anesthesiol 2008, 20:188-192.

23. Hoff RG, Rinkel GJ, Verweij BH, Algra A, Kalkman CJ: Pulmonary edema and blood volume after aneurysmal subarachnoid hemorrhage: a prospective observational study. Crit Care 2010, 14:R43.

24. Broessner G, Helbok R, Lackner P, Mitterberger M, Beer R, Engelhardt K, Brenneis C, Pfausler B, Schmutzhard E: Survival and long-term functional outcome in 1,155 consecutive neurocritical care patients. Crit Care Med 2007, 35:2025-2030
25. Kiphuth IC, Schellinger PD, Kohrmann M, Bardutzky J, Lucking H, Kloska S, Schwab S, Huttner HB: Predictors for good functional outcome after neurocritical care. Crit Care 2010, 14:R136.

26. Kiphuth IC, Kohrmann M, Kuramatsu JB, Mauer C, Breuer L, Schellinger PD, Schwab S, Huttner HB: Retrospective agreement and consent to neurocritical care is influenced by functional outcome. Crit Care 2010, 14:R144.

27. Diedler J, Sykora M, Hahn P, Heerlein K, Scholzke MN, Kellert L, Bosel J, Poli S, Steiner T: Low hemoglobin is associated with poor functional outcome after non-traumatic, supratentorial intracerebral hemorrhage. Crit Care 2010, 14:R63.

28. Mondello S, Papa L, Buki A, Bullock MR, Czeiter E, Tortella FC, Wang KK, Hayes RL: Neuronal and glial markers are differently associated with computed tomography findings and outcome in patients with severe traumatic brain injury: a case control study. Crit Care 2011, 15:R156.

29. Chen XH, Meaney DF, Xu BN, Nonaka M, McIntosh TK, Wolf JA, Saatman KE, Smith DH: Evolution of neurofilament subtype accumulation in axons following diffuse brain injury in the pig. J Neuropathol Exp Neurol 1999, 58:588-596.

30. Kleindienst A, Hesse F, Bullock MR, Buchfelder M: The neurotrophic protein S100B: value as a marker of brain damage and possible therapeutic implications. Prog Brain Res 2007, 161:317-325.

31. Tolentino PJ, DeFord SM, Notterpek L, Glenn CC, Pike BR, Wang KK, Hayes RL: Up-regulation of tissue-type transglutaminase after traumatic brain injury. J Neurochem 2002, 80:579-588.

32. Earle $S A$, Proctor $K G$, Patel MB, Majetschak M: Ubiquitin reduces fluid shifts after traumatic brain injury. Surgery 2005, 138:431-438.

33. Dolinak D, Reichard R: An overview of inflicted head injury in infants and young children, with a review of beta-amyloid precursor protein immunohistochemistry. Arch Pathol Lab Med 2006, 130:712-717.

34. Guzel A, Karasalihoglu S, Aylanç H, Temizöz O, Hiçdönmez T: Validity of serum tau protein levels in pediatric patients with minor head trauma. Am J Emerg Med 2010, 28:399-403.

35. Dong $X Q$, Yang $S B$, Zhu FL, Lv QW, Zhang GH, Huang HB: Resistin is associated with mortality in patients with traumatic brain injury. Crit Care 2010, 14:R190.

doi:10.1186/cc10423

Cite this article as: Scalfani MT, Diringer MN: Year in review 2010: Critical Care - neurocritical care. Critical Care 2011, 15:237. 